variations of sign in (2); for $x=-\infty$ there are $s$, thus $H_{n}(x)=0$ has at least $\sigma$ real roots.

We now consider the general case that the sequence (2) has common roots. With Weber we may dispose of this case as follows. Suppose e.g. that $H_{k}, H_{k-1}$ have common roots. We vary the terms $a_{i j}$ of $H_{k}$ not in $H_{k-1}$ by small amounts numerically less than some $\eta$, so that $H_{k}, H_{k-1}$ do not have common roots.

In this way we may replace (2) by another sequence

$$
K_{n}, K_{n-1}, K_{n-2}, \cdots, K_{1}, K_{0}=1
$$

no two of which have a common root. The roots of $K_{n}=0$ differ from those of $H_{n}=0$ by an amount as small as we please, for sufficiently small $\eta$, moreover the signs of corresponding elements of the sequences (2), (5) are the same for an $x$ for which no element of (2) vanishes. As Theorem I holds for (5), it must hold for (2).

THEOREM II. The roots of the secular equations are all real. For in this equation $s=0$; hence $\sigma=n$.

YALE UNIVERSITY

\title{
A GENERALIZED TWO-DIMENSIONAL POTENTIAL PROBLEM
}

\section{BY J. R. CARSON}

It may be shown that the solution of the problem of electromagnetic wave propagation along a system of straight parallel conductors can be reduced to the solution* of two subsidiary problems: (1) a well known problem in twodimensional potential theory; and (2) a generalization of the two-dimensional potential problem which is believed to be novel. The generalized problem is believed to possess suffi-

\footnotetext{
* Subject to certain restrictions to be discussed in a forthcoming paper.
} 
cient mathematical interest, in addition to its technical importance, to justify its publication in this note.

The two-dimensional potential problem will first be stated in order to exhibit its relation to the generalized problem:

Let there be $n$ closed curves in the $x y$-plane and let a function $\Phi(x, y)$ satisfy Laplace's equation in two dimensions everywhere outside these curves; thus

$$
\left(\frac{\partial^{2}}{\partial x^{2}}+\frac{\partial^{2}}{\partial y^{2}}\right) \Phi=0 .
$$

On the $j$ th curve $(j=1,2, \cdots, n)$, let

$$
\frac{\partial}{\partial \tau} \Phi=0, \quad \int \frac{\partial}{\partial n} \Phi d \tau=Q_{j}, \quad(j=1,2, \cdots, n) .
$$

In equations (2), $n$ and $\tau$ are the vectors normal and tangential to the $j$ th curve respectively and $Q_{j}$ is a specified real constant.

The determination of the potential function $\Phi$ in terms of $Q_{1}, \cdots, Q_{n}$ and the geometry of the field is a well known problem in the theory of the potential for which very general methods of solution are available. The solution is of the form

$$
\Phi=\phi_{1}(x, y) Q_{1}+\cdots+\phi_{n}(x, y) Q_{n}
$$

the coefficients $\phi_{1}, \cdots, \phi_{n}$, themselves two-dimensional potentials, being determined by the geometry of the field.

The generalized problem, which is the subject of this note, may be stated as follows.

Let there be $n$ closed curves in the $x y$-plane, and let a function $F$ satisfy the equation

$$
\left(\frac{\partial^{2}}{\partial x^{2}}+\frac{\partial^{2}}{\partial y^{2}}\right) F=0
$$

everywhere outside these curves. 
Inside the $j$ th curve let a function $E_{i}$ satisfy the equation

$$
\left(\frac{\partial^{2}}{\partial x^{2}}+\frac{\partial^{2}}{\partial y^{2}}\right) E_{j}=\nu_{j}^{2} E_{j}, \quad(j=1,2, \cdots, n),
$$

where $\nu_{i}^{2}$ is a given parameter, in general complex.

On the $j$ th curve let $F$ and $E_{j}$ be connected by the equations

(6) $\frac{\partial}{\partial \tau} F=-\frac{\partial}{\partial \tau} E_{j}, \frac{\partial}{\partial n} F=-\mu_{j} \frac{\partial}{\partial n} E_{i},(j=1,2, \cdots, n)$.

Here, as above, $n$ and $\tau$ are the vectors normal and tangential to the $j$ th curve respectively, and $\mu_{j}$ is a given real parameter.

Finally

$$
\int \frac{\partial}{\partial n} E_{j} d \tau=-\int \frac{\partial}{\partial n} F d \tau=J_{i}, \quad(j=1,2, \cdots, n),
$$

where $J_{j}$ is a specified constant, in general complex. The integration is carried around the contour of the $j$ th curve.

We require the determination of $F$ outside the curves and $E_{j}$ inside the $j$ th curve in terms of $\lambda_{i}, \mu_{i}, \nu_{j}, J_{j}$ and the geometry of the field, in the form

$$
\begin{gathered}
F=f_{1}(x, y) J_{1}+\cdots+f_{n}(x, y) J_{n}, \\
E_{i}=e_{j 1}(x, y) J_{1}+\cdots+e_{j n}(x, y) J_{n} .
\end{gathered}
$$

This problem has been solved for the practically important case where all the curves are circles by expanding $E_{i}$ as a Fourier-Bessel series and identifying the constants of the expansion by a process of successive approximations. As yet, however, no serious study has been made of the general problem. Its analogy with the potential problem suggests, however, that extensions of the well known methods there available should be successful in the solution of the generalized problem.

American Telegraph and Telephone Company 